\title{
Conditional viability for Impulse Differential Games
}

Jean-Pierre Aubin and Nicolas Seube

presented by

Alexandre Bayen
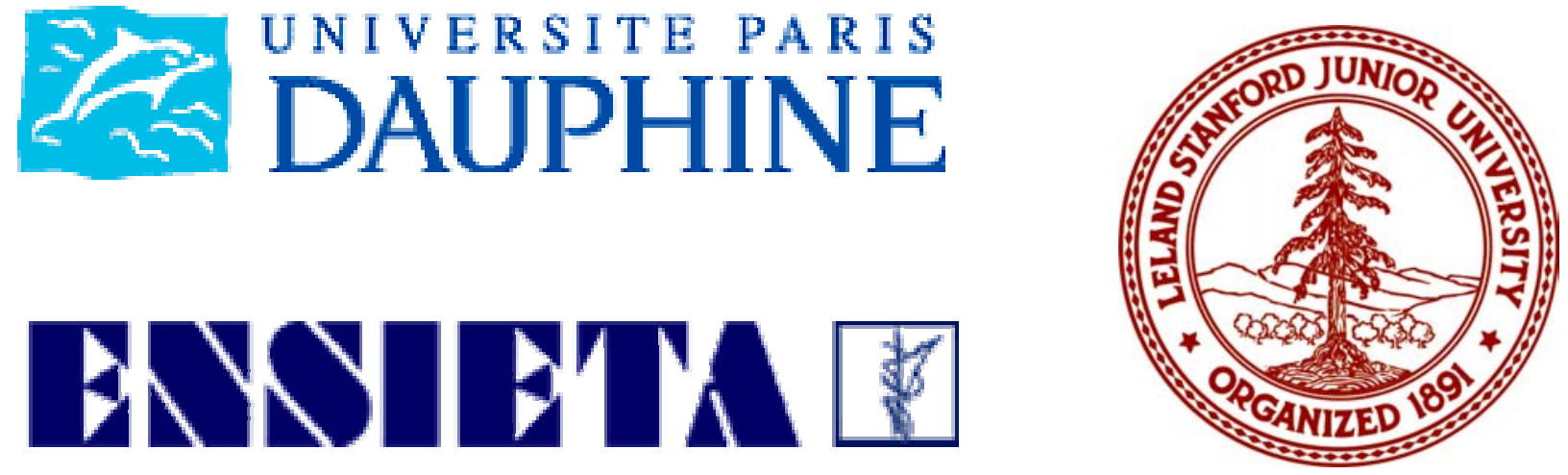

Conference on Decision and Control, Las Vegas, Dec. $13^{\text {th }}, 2002$ 


\section{$1957 . .$. Isaacs writes Differential Games}

DIFFERENTIAL GAMES

\section{RUFUS ISAACS}

The game of two cars... The homicidal chauffeur...

$$
\frac{d}{d t}\left(\begin{array}{l}
x \\
y \\
\psi
\end{array}\right)=\mathbf{f}(\mathbf{x}, u, d)=\left(\begin{array}{c}
-v+v \cos \psi+u y \\
v \sin \psi-u x \\
d-u
\end{array}\right)
$$

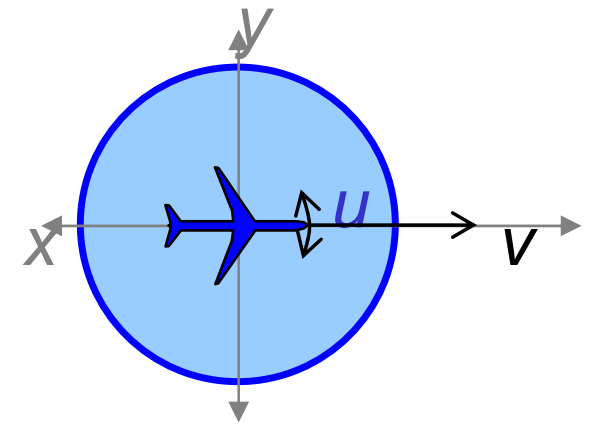

'evader' (control)

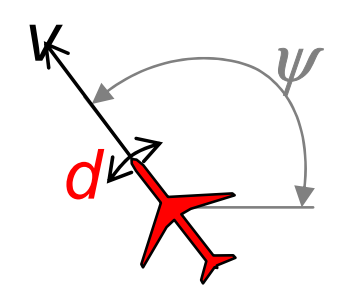

'pursuer'

(disturbance)

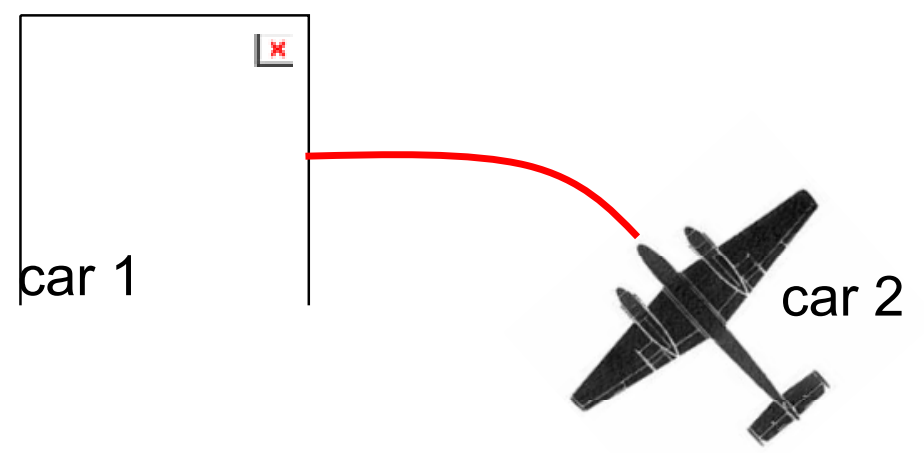




\section{$1971 \ldots$ people are still working on it}

THE HOMICIDAL CHAUFFEUR - A DIFFERENTIAL GAME

\section{A DISSERTATION}

SUBMITTED TO THE DEPARTMENT OF AERONAUTICS AND ASTRONAUTICS AND THE COMMITTEE ON GRADUATE STUDIES

OF STANFORD UNIVERSITY

IN PARTIAL FULFILLMENT OF THE REQUIREMENTS

FOR THE DEGREE OF

DOCTOR OF PHILOSOPHY

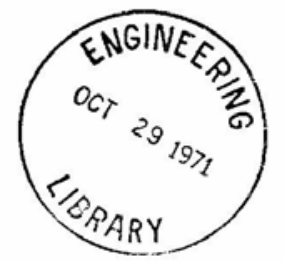

By

Antony Willits Merz

March 1971

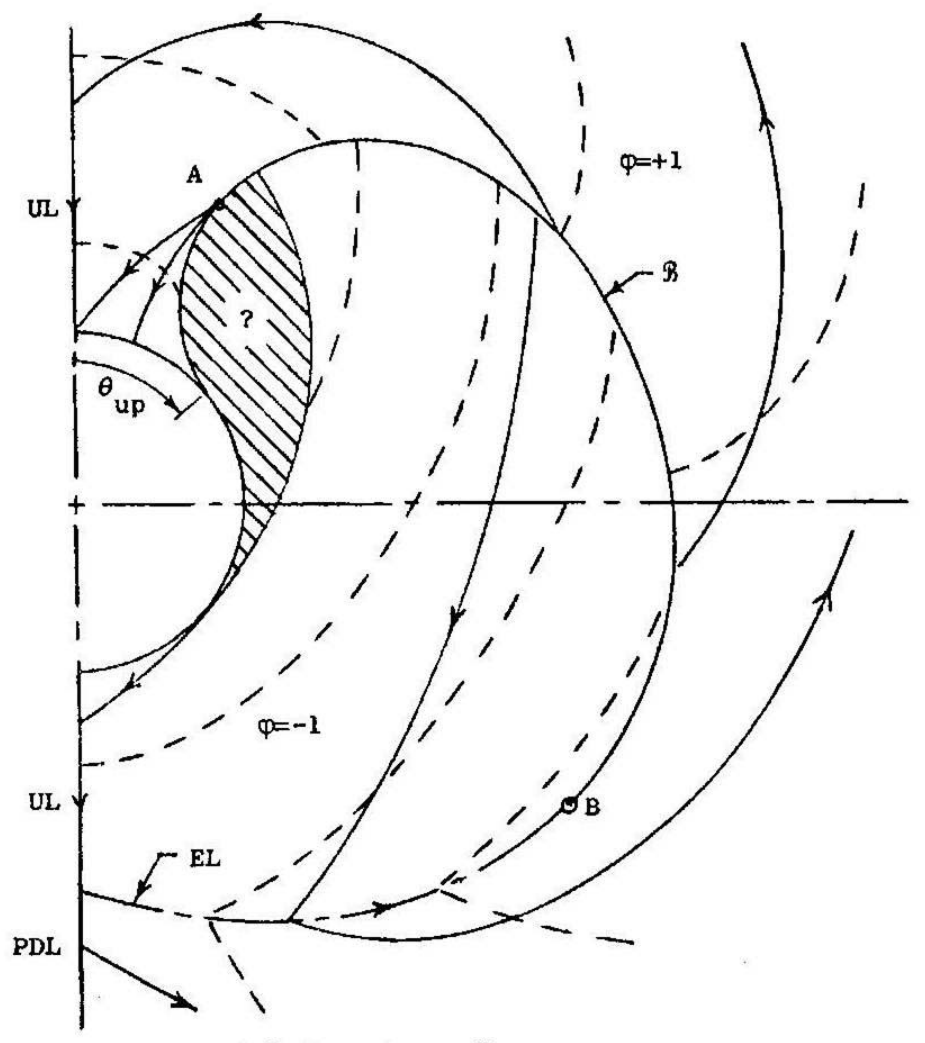

(b) Barriers Open

FIGURE 2.5. Trajectories and Isochrones in the Classical Game 

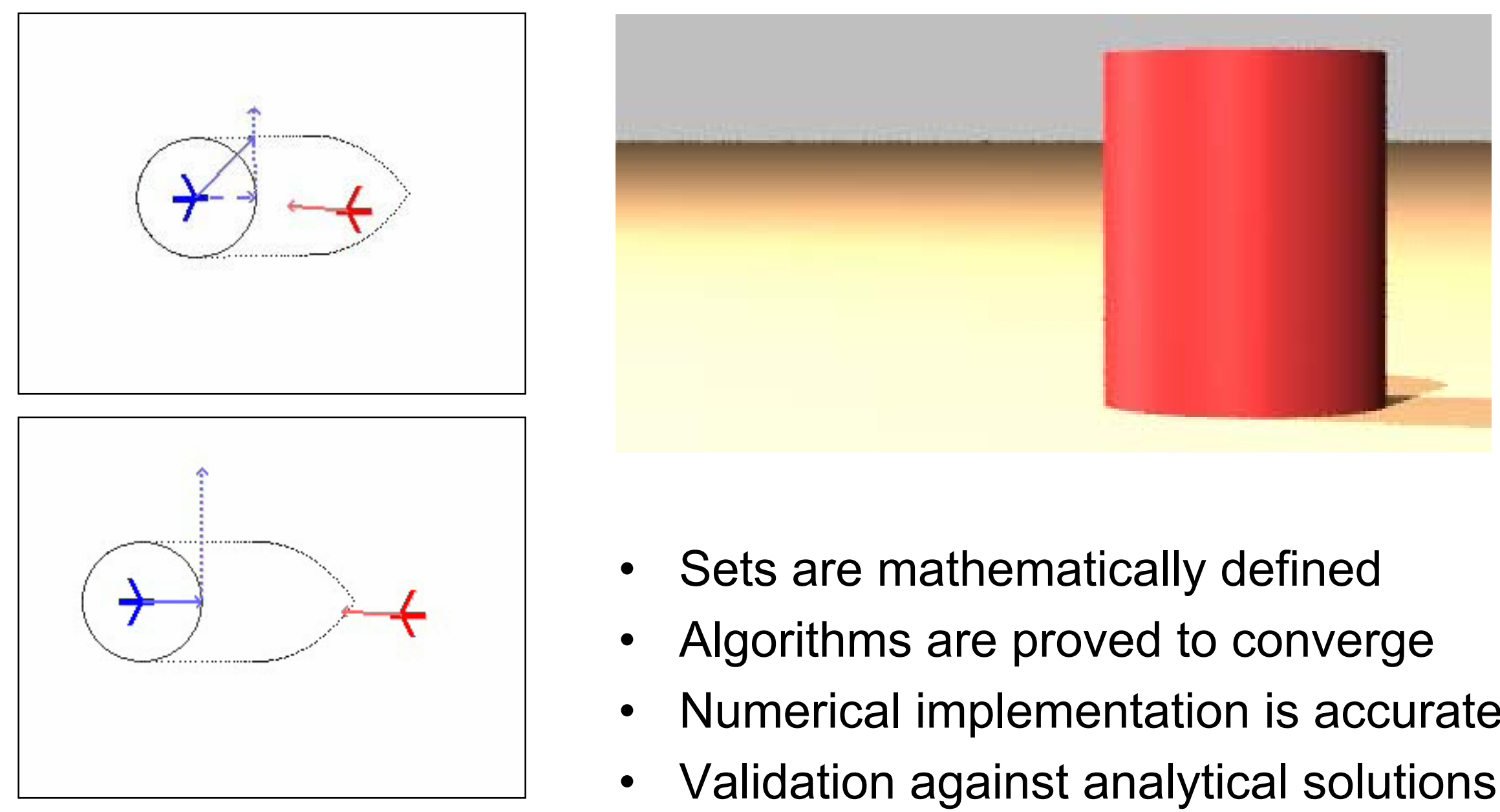

- Sets are mathematically defined

- Algorithms are proved to converge

- Numerical implementation is accurate

- Validation against analytical solutions

[Mitchell, Bayen, Tomlin subm. IEEE TAC 2001] 


\section{$2002 \ldots$ forty five years after...}

- 1957: Isaacs links differential games to the Hamilton-Jacobi equation.

- 1982: Crandall, Evans and Lions discover the viscosity solution of Hamilton-Jacobi equation.

- 1991: Aubin defines the viability kernel and capture basins mathematically.

- 1994: First numerical computations of reachable sets: SaintPierre, Falcone.

- 1997: Link between the static Hamilton-Jacobi equation and viability: Frankowska, Cardaliaguet, Quincampoix, Saint-Pierre.

- 2000: Level set methods are used to compute accurately reachable sets: Tomlin, Sastry, Lygeros, Mitchell.

- 2002: Hyperbolic Hamilton-Jacobi equation is linked to reachability and viability: Mitchell, Bayen, Tomlin. 


\section{Outline}

1. A short history of differential games

2. Impulse evolutionary systems

1. Runs

2. Impulse viability kernel

3. The swimmer problem

3. Impulse evolutionary games

1. Impulse conditional viability kernel

2. Impulse discriminating kernel

3. Tax pollution

4. Conclusion 


\section{Evolutionary systems}

\section{$\in \hookrightarrow \quad \subset C(0 . \infty ; X)$}
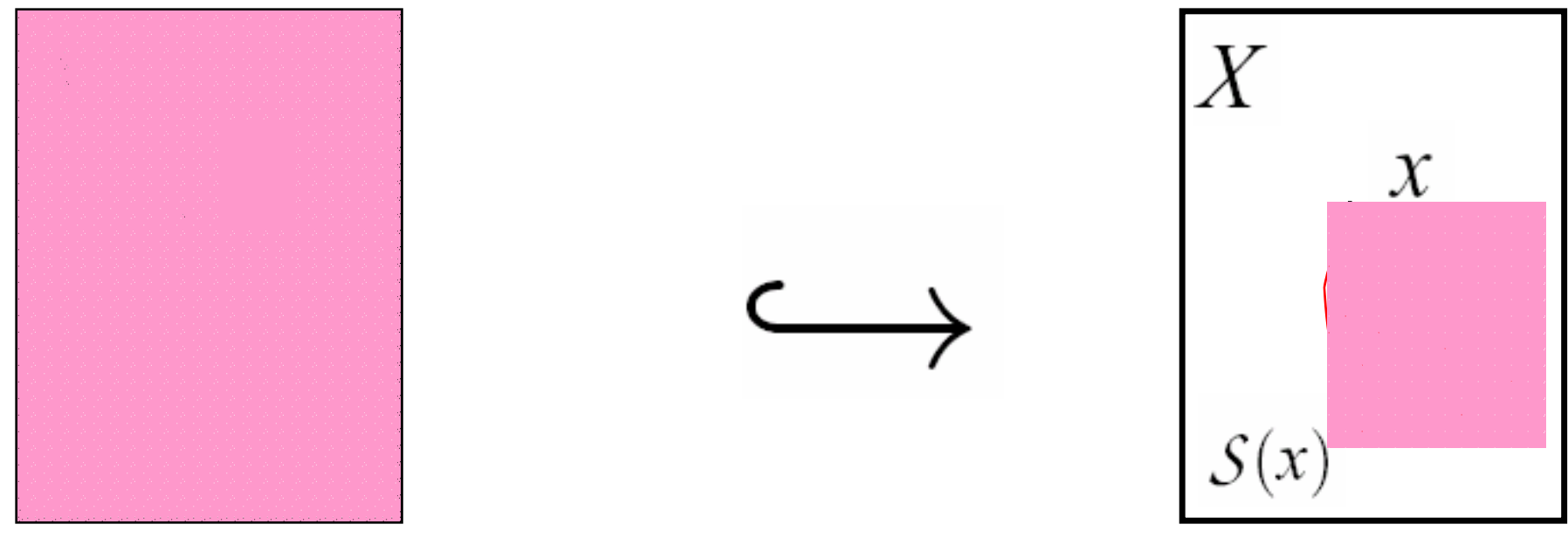

Example: dynamical system

$$
\begin{aligned}
& \left\{\begin{array}{l}
\dot{x}(t)=f(x(t), u(t)) \\
x(0)=x
\end{array}\right. \\
& \left\{\begin{array}{l}
\dot{x}(t) \in F(x(t))=\{f(x(t), u) \mid u \in U\} \\
x(0)=x
\end{array}\right.
\end{aligned}
$$




\section{Evolutionary systems}

$$
x \in X \hookrightarrow S(x) \subset \mathcal{C}(0 . \infty ; X)
$$

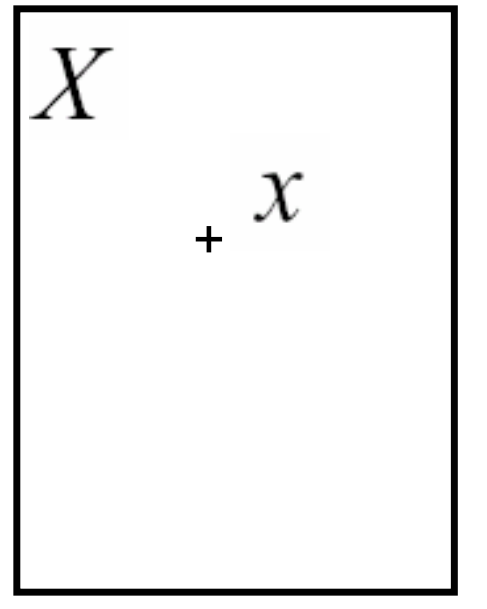

Space $X$

Point $x$

Set of trajectories $S(x)$

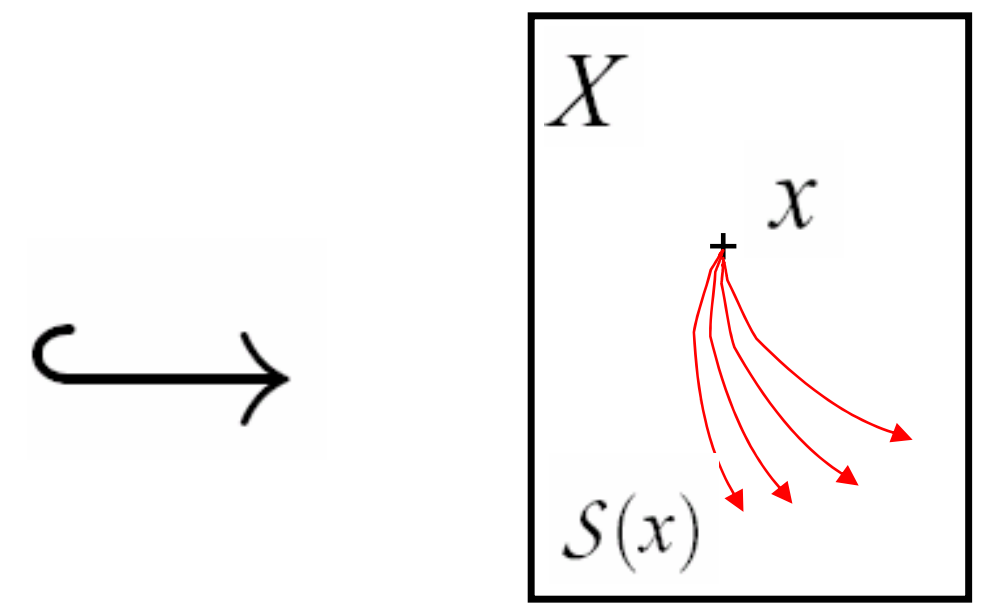

Definition:

$x \in X \hookrightarrow \mathcal{S}(x) \subset \mathcal{C}(0, \infty ; X)$

is called an evolutionary system 


\section{Runs of impulse evolutionary systems}

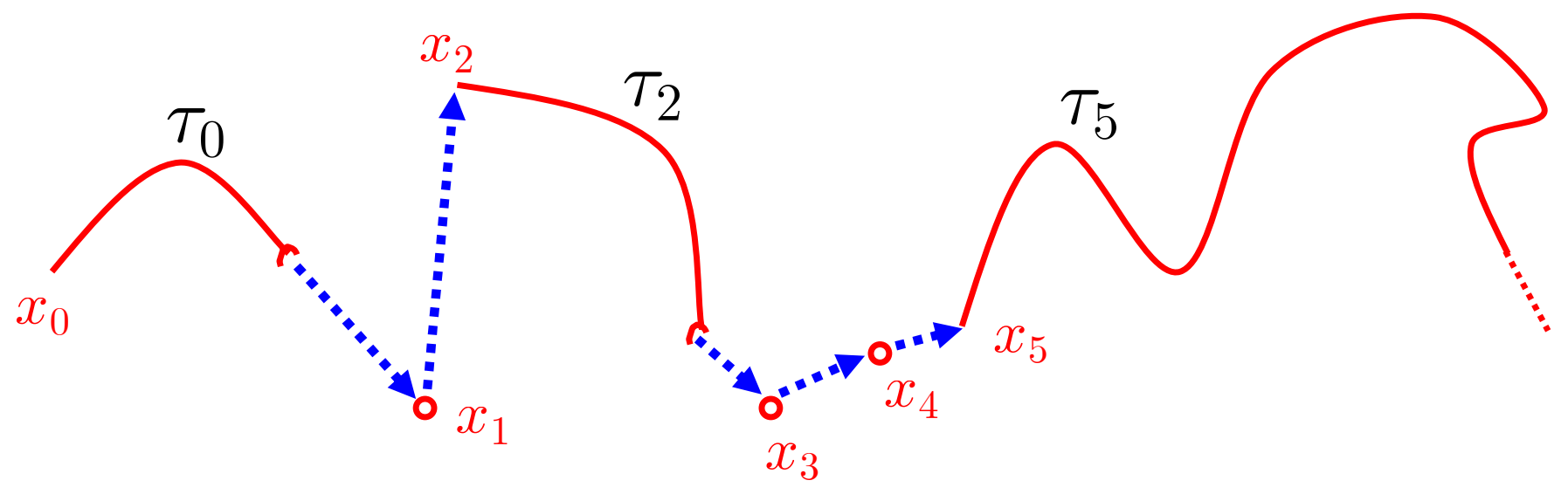

$\vec{x}(\cdot):=\left(\tau_{n}, x_{n}, x_{n}(\cdot)\right)_{n \geq 0} \in\left(\mathbf{R}_{+} \times X\right)^{\mathbf{N}} \times \prod_{n=0}^{\infty} \mathcal{C}\left(0, \tau_{n} ; X\right)$

Pieces of trajectories, with jumps

$\Phi: X \hookrightarrow X \quad$ Reset function

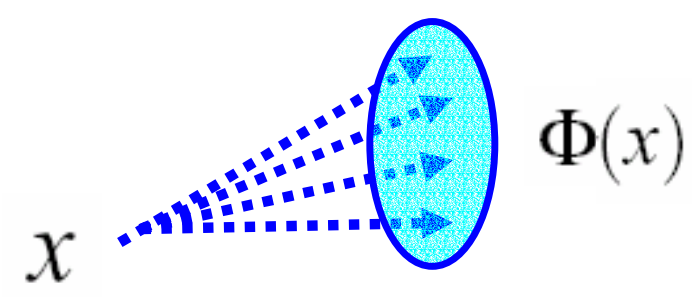




\section{Impulse viability kernel}

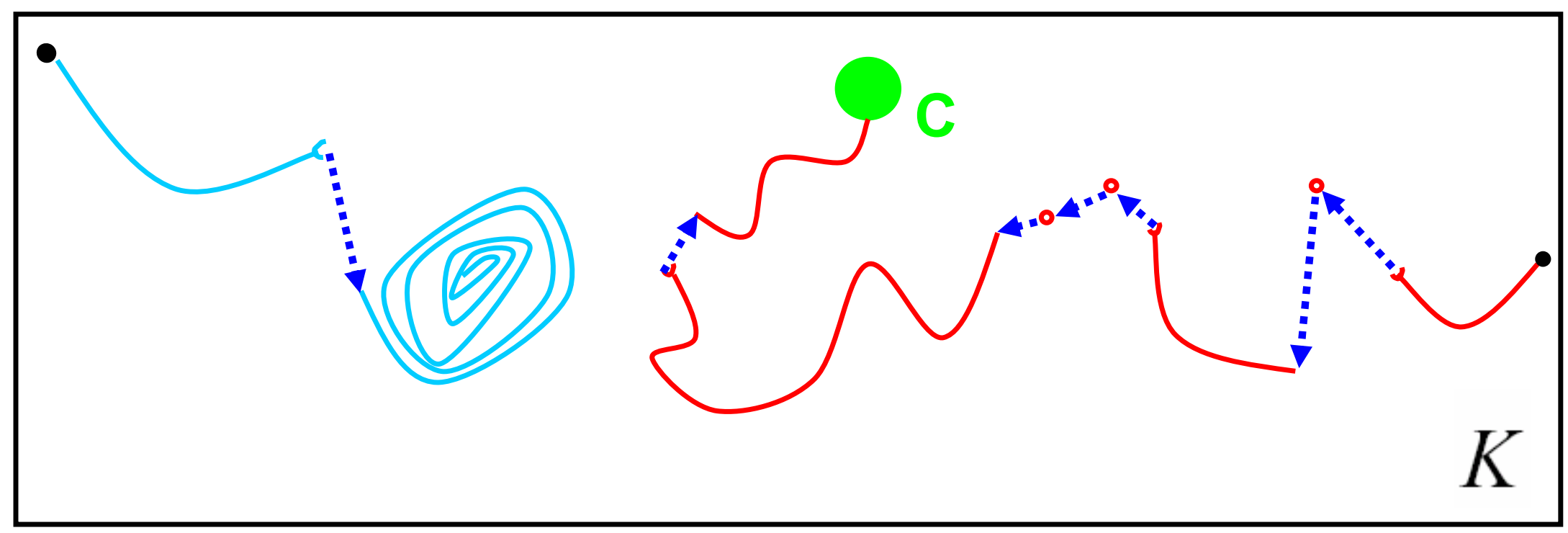

Definition 2.1 Let $K$ and $C \subset K$ be two subsets. The impulse viability kernel of $K$ with target $C \subset K$

$$
\operatorname{ImpViab}(K, C):=\operatorname{ImpViab}_{(S, \Phi)}(K, C)
$$

of $K$ with target $C \subset K$ under the impulse evolutionary system $(\mathcal{S}, \Phi)$ is the subset of initial states $x \in K$ from which starts at least one run viable in $K$ forever or until it reaches the target $C$ in finite time. 


\section{Impulse viability kernel}

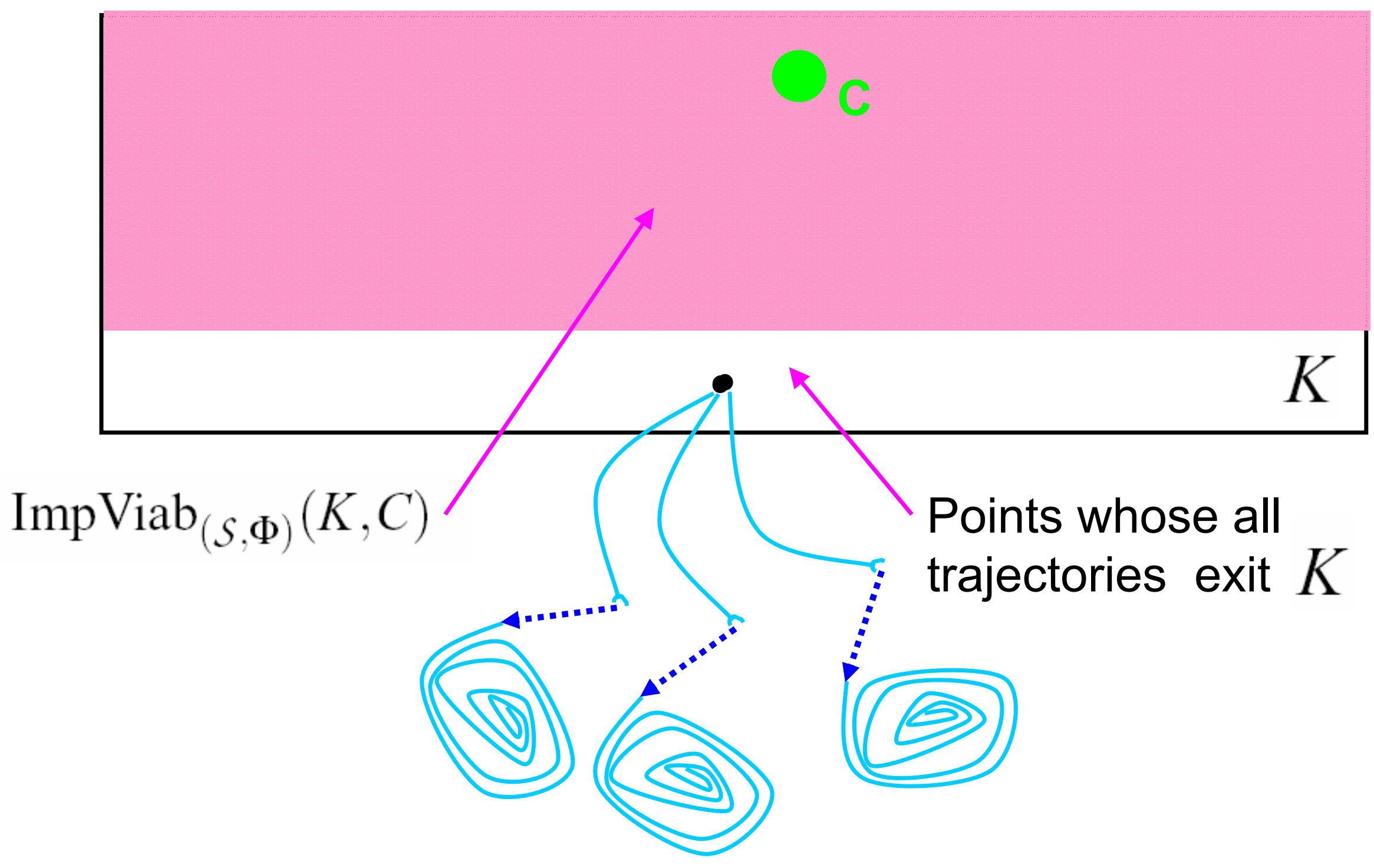




\section{Impulse viability kernel}

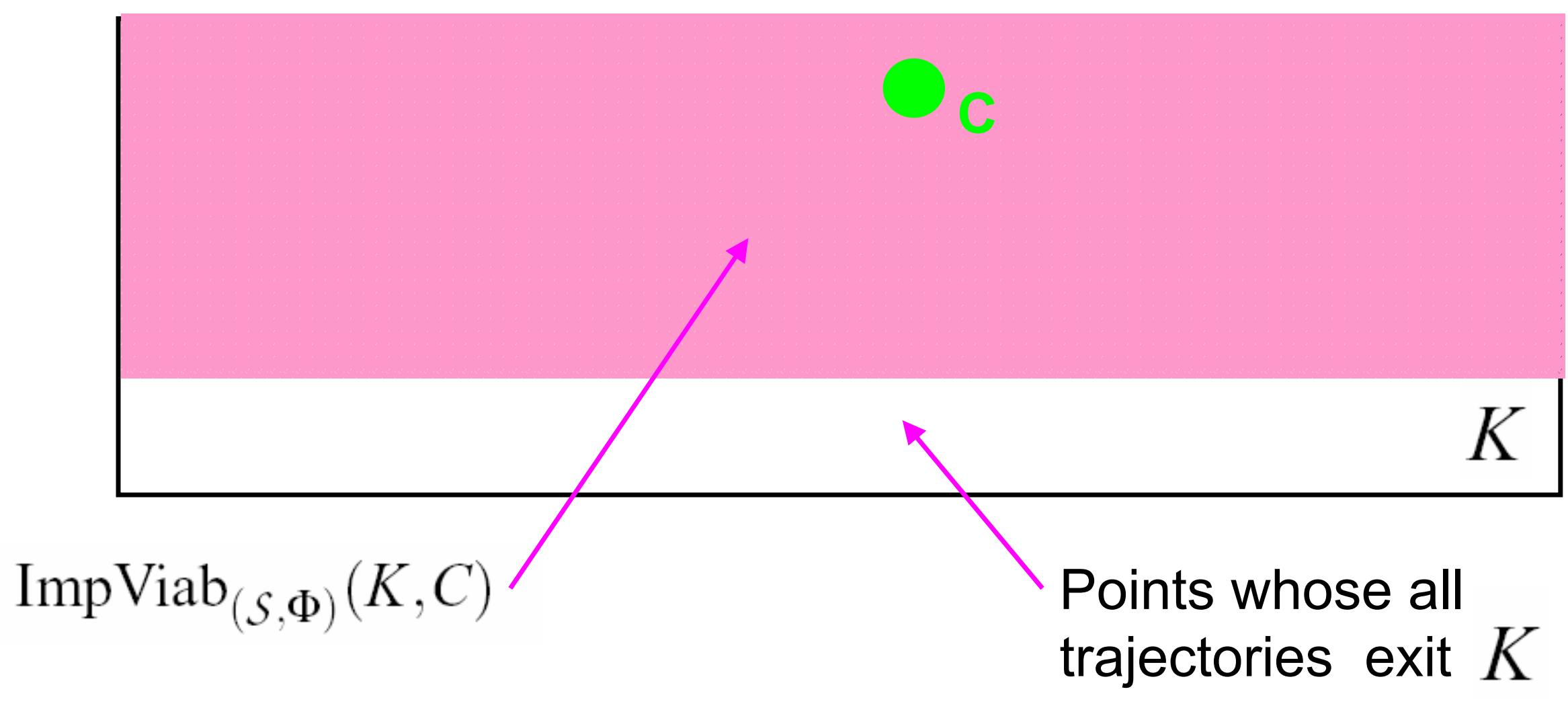

$C \subset \operatorname{ImpViab}_{(\mathcal{S}, \Phi)}(K, C) \subset K$

if $C_{1} \subset C_{2}$ and $Q_{1} \subset Q_{2}$

then $\operatorname{ImpViab}_{(S, \Phi)}\left(Q_{1}, C_{1}\right) \subset \operatorname{ImpViab}_{(S, \Phi)}\left(Q_{2}, C_{2}\right)$ 


\section{Properties of the impulse viability kernel}

\section{$\operatorname{ImpViab}_{(\mathcal{S}, \Phi)}(K, C)$ is:}

1. the largest subset $D$ satisfying $C \subset D \subset K$ and $D \subset$ $\operatorname{ImpViab}_{(\mathcal{S}, \Phi)}(D, C)$,

2. the smallest subset $D$ satisfying $C \subset D \subset K$ and $\operatorname{ImpViab}_{(\mathcal{S}, \Phi)}(K, D) \subset D$,

3. the unique subset $D$ satisfying $C \subset D \subset K$ and

$$
D=\operatorname{ImpViab}_{(\mathcal{S}, \Phi)}(K, D)=\operatorname{ImpViab}_{(\mathcal{S}, \Phi)}(D, C)
$$




\section{Example: the Zermelo problem}
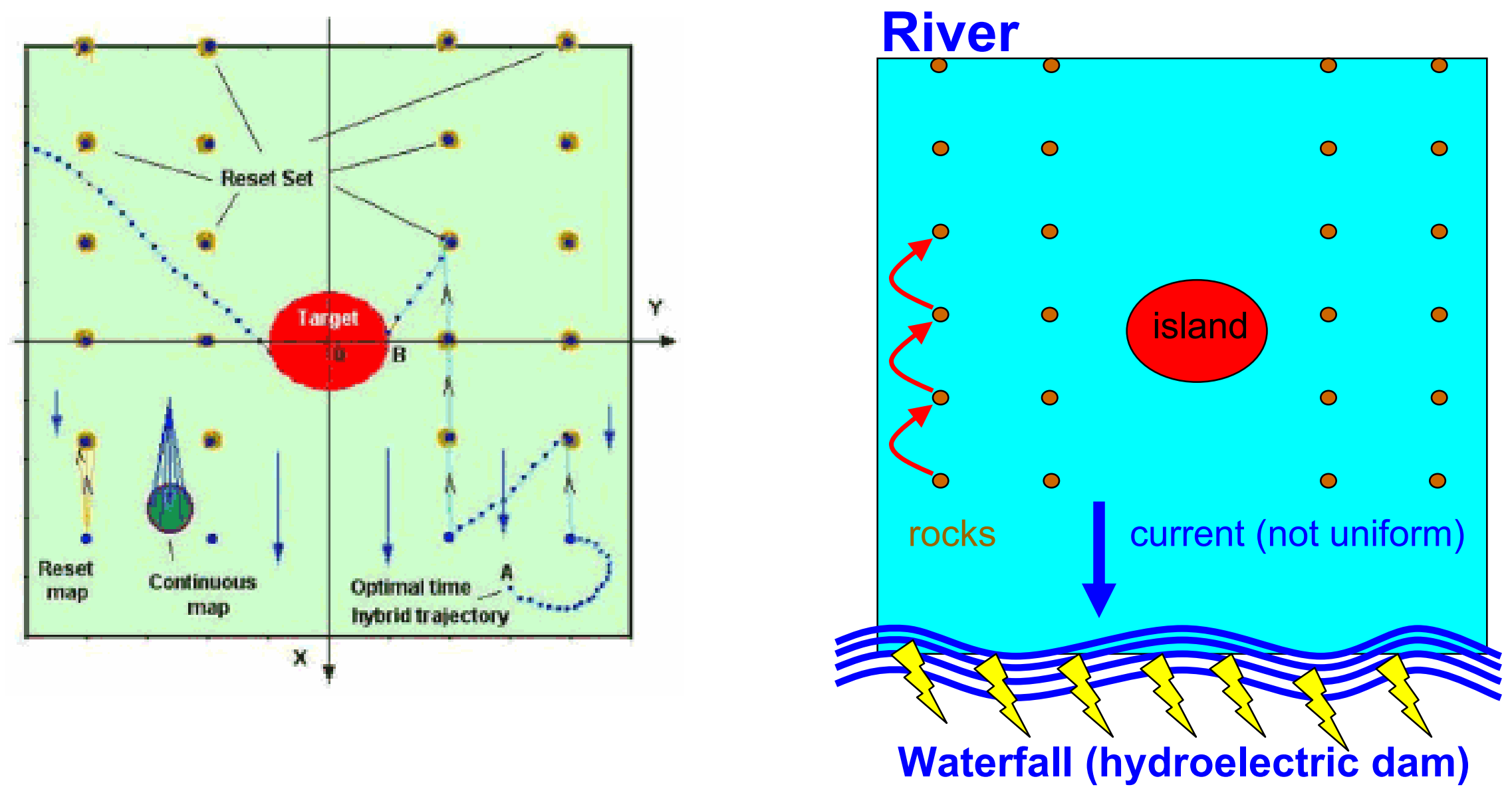

[Saint-Pierre, 2002] 


\section{Example: the Zermelo problem}

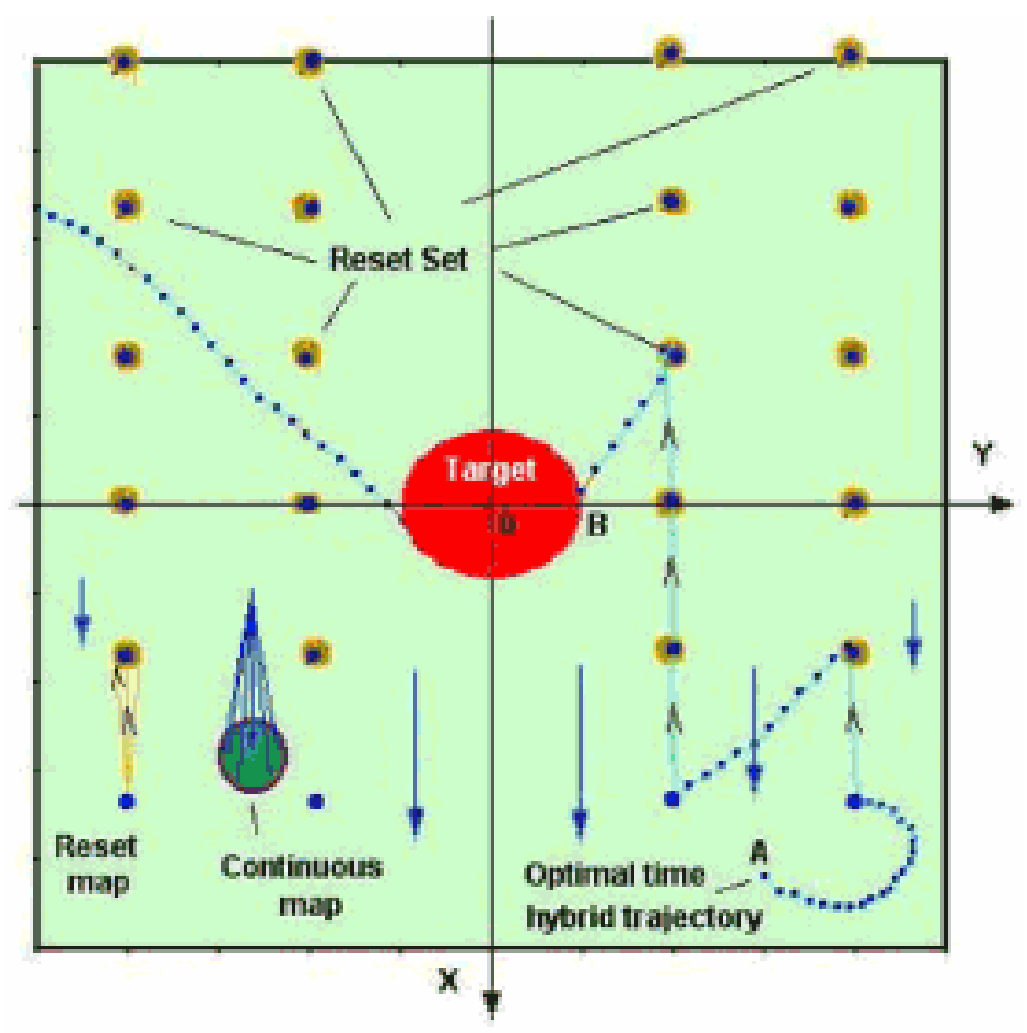

In this region, the swimmer can:

1) swim / jump / swim / jump ... forever 2) swim / jump /swim ... reach the island

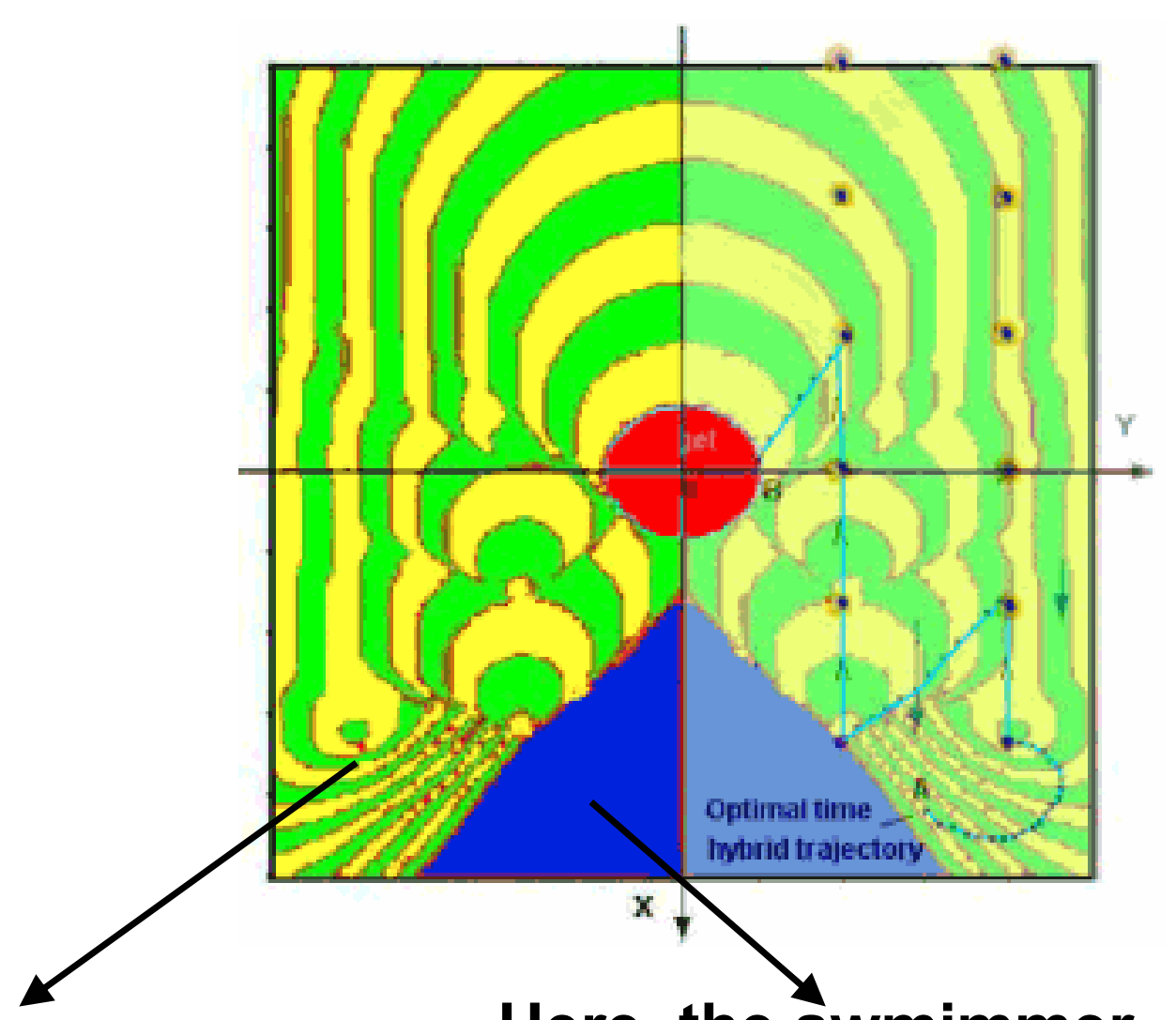

Here, the swmimmer goes into the waterfall (and dies)

[Saint-Pierre, HSCC 2002] 


\section{Outline}

1. A short history of differential games

2. Impulse evolutionary systems

1. Runs

2. Impulse viability kernel

3. The swimmer problem

3. Impulse evolutionary games

1. Impulse conditional viability kernel

2. Impulse discriminating kernel

3. Tax pollution

4. Conclusion 


\section{Evolutionary games}
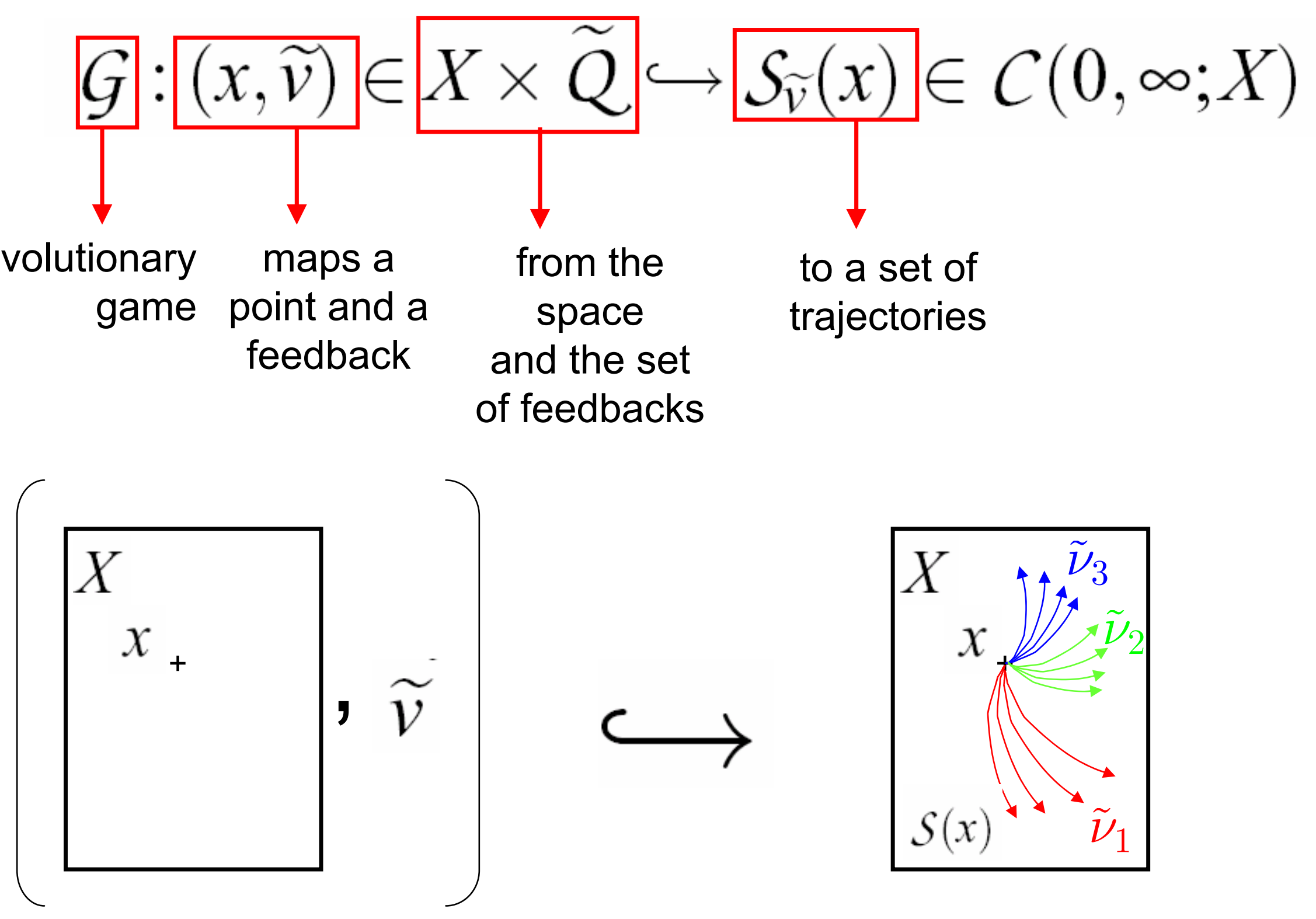


\section{Example: differential games}

\section{Differential system:}

$$
\left\{\begin{array}{l}
\left.x^{\prime}(t) \in F(x(t), u(t), v(t))\right) \\
u(t) \in P(x(t)) \\
v(t) \in Q(x(t))
\end{array}\right.
$$

Evolutionary game: $(x, \widetilde{v}) \hookrightarrow \mathcal{S}_{\widetilde{v}}(x)$ where

$\mathcal{S}_{\widetilde{v}}(x)$ is the set of solution of:

$$
x^{\prime}(t) \in F(x(t), P(x(t)), \widetilde{v}(x(t)))
$$




\section{Conditional Impulse Viability Kernel}

$\operatorname{CondImpViab}_{\mathcal{G}}(K, C):=\bigcap \operatorname{ImpViab}_{\widetilde{v}}(K, C)$

For all feedback $\widetilde{v}$

The set of points which stay in $\mathrm{K}$ forever, or reach C in final time whatever $\widetilde{v}$ decides to play 


\section{Impulse discriminating Viability Kernel}

For an evolutionary game $(\mathcal{S}, \Phi)$

And two subsets $K$ and $C \subset K$.

The largest subset $D$ of $K$ containing $C$, conditionally viable in $K$ outside $C$ under the impulse evolutionary game $(x, \widetilde{v}) \hookrightarrow \mathcal{S}_{\widetilde{v}}(x)$ is the impulse discriminating kernel of $K$ with target $C$ 


\section{Continuous example: tax-pollution}

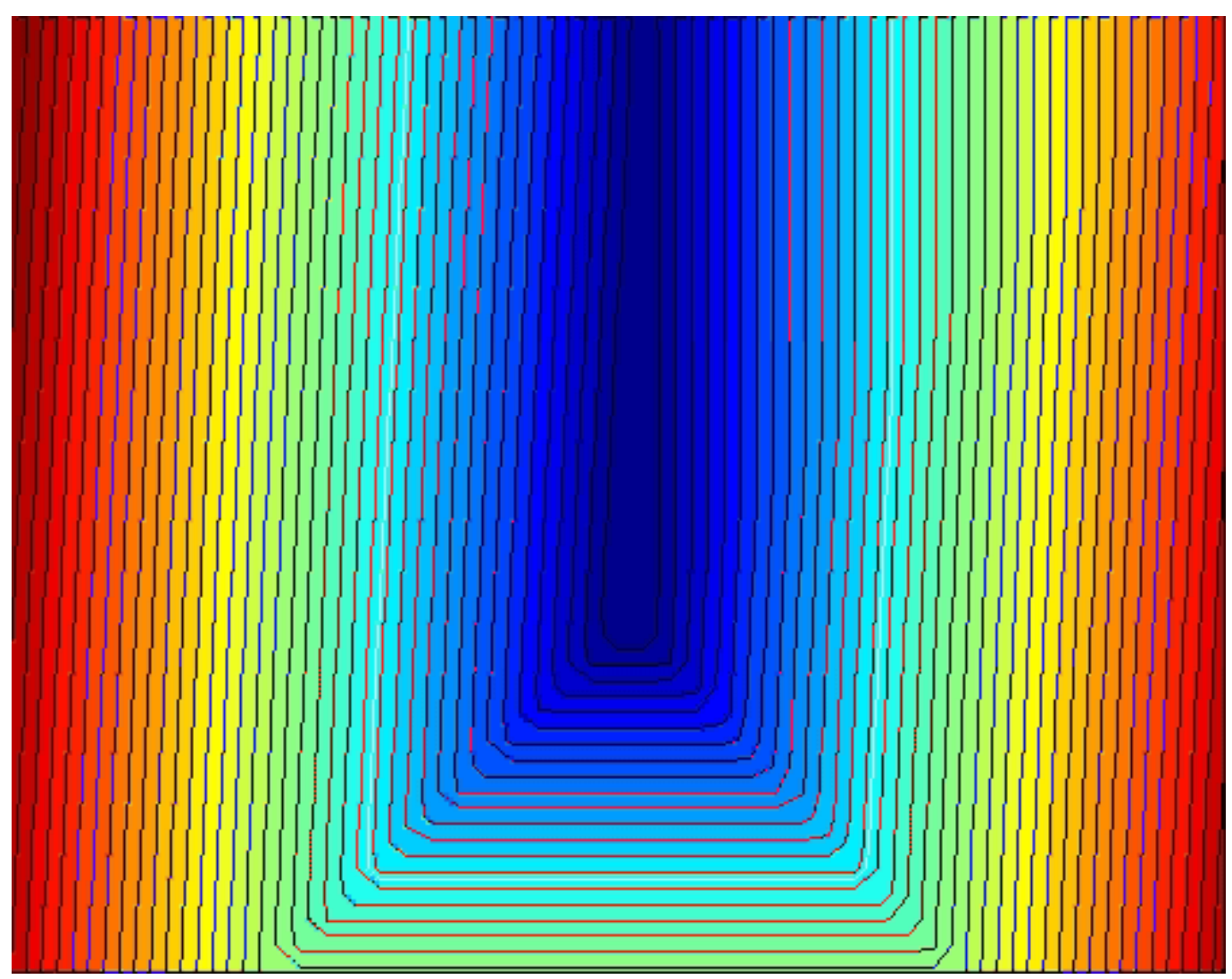

$\begin{array}{ll}\dot{x}=u x-p & u \in[0.5,1.2] \\ \dot{p}=v & v \in[-0.5,0.5]\end{array} \quad(x, p) \in[0.2,2] \times R^{+}$

[Bayen Tomlin, CDC 2001] 


\section{Theorem The conditional viability kernel}

CondImpViab $_{\mathcal{G}}(K, C)$ of a subset $K$ with target $C \subset K$ is

the smallest subset $D \in \mathcal{D}(K, C)$ satisfying

$$
\operatorname{CondImpViab}_{\mathcal{G}}(K, D) \subset D
$$

and actually is a fixed point

$$
\operatorname{CondImpViab}_{\mathcal{G}}(K, C)=\bigcap_{\widetilde{v}} \operatorname{ImpViab}_{\widetilde{v}}\left(K, \operatorname{CondImpViab}_{\mathcal{G}}(K, C)\right)
$$

Unfortunately, the conditional impulse viability kernel CondImpViab $\operatorname{Gi}_{\mathcal{G}}(K, C)$ of $K$ outside the target $C \subset$ $K$ is not itself necessarily conditionally viable outside the target $C$, i.e., it is not a fixed point of the map $K \mapsto$ CondImpViab $_{\mathcal{G}}(K, C)$. 


\section{Properties of the Discriminating Kernel}

$\operatorname{ImpDisc}(K, C)$ is the largest fixed point of the map

$K \in \mathcal{D}(K, C) \mapsto \operatorname{CondImpViab}_{\mathcal{G}}(K, C)$

$\operatorname{ImpDisc}(\operatorname{ImpDisc}(K, C), C)=\operatorname{ImpDisc}(K, C)$

Proposition Let us consider an evolutionary game $(x, \widetilde{v}) \hookrightarrow \mathcal{S}_{\widetilde{v}}(x)$

Then $C \mapsto \operatorname{ImpDisc}(K, C)$ is increasing

$\operatorname{ImpDisc}(K, \operatorname{ImpDisc}(K, C))=\operatorname{ImpDisc}(K, C)$ 


\section{Conclusions}

- Viability theory introduces new concepts:

Set valued dynamics, the viability kernel, the capture basin

They can be use to define reachable sets of evolutionary systems

- The main characterization theorems

Enables to prove the existence, maximality and uniqueness of the viability kernel, and to compute them.

- The representation of these sets is algebraic

- Open research directions: algorithms, convergence 


\section{Tomorrow's talk... $\quad$ Fr-P07-5 5:40pm}
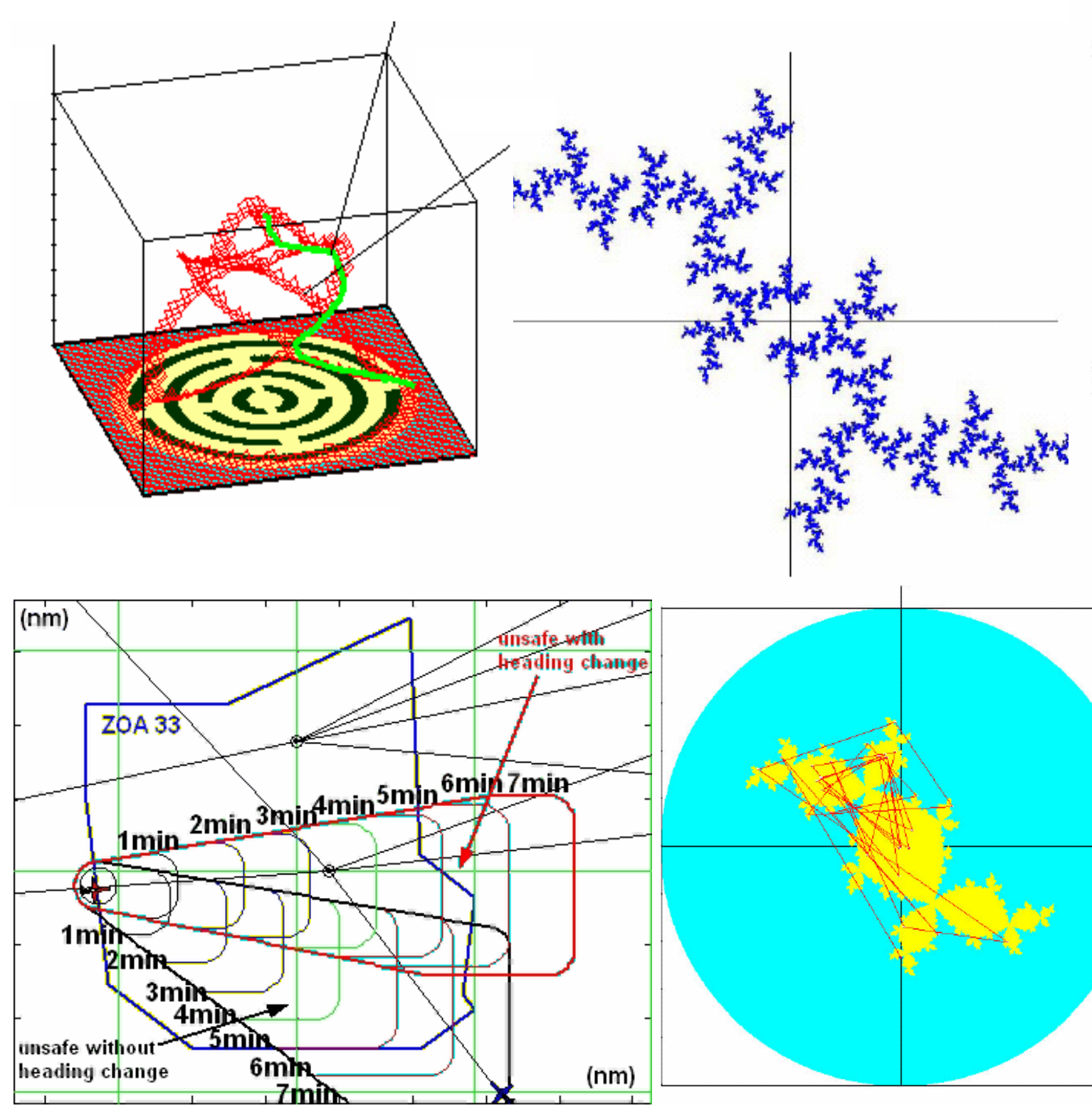
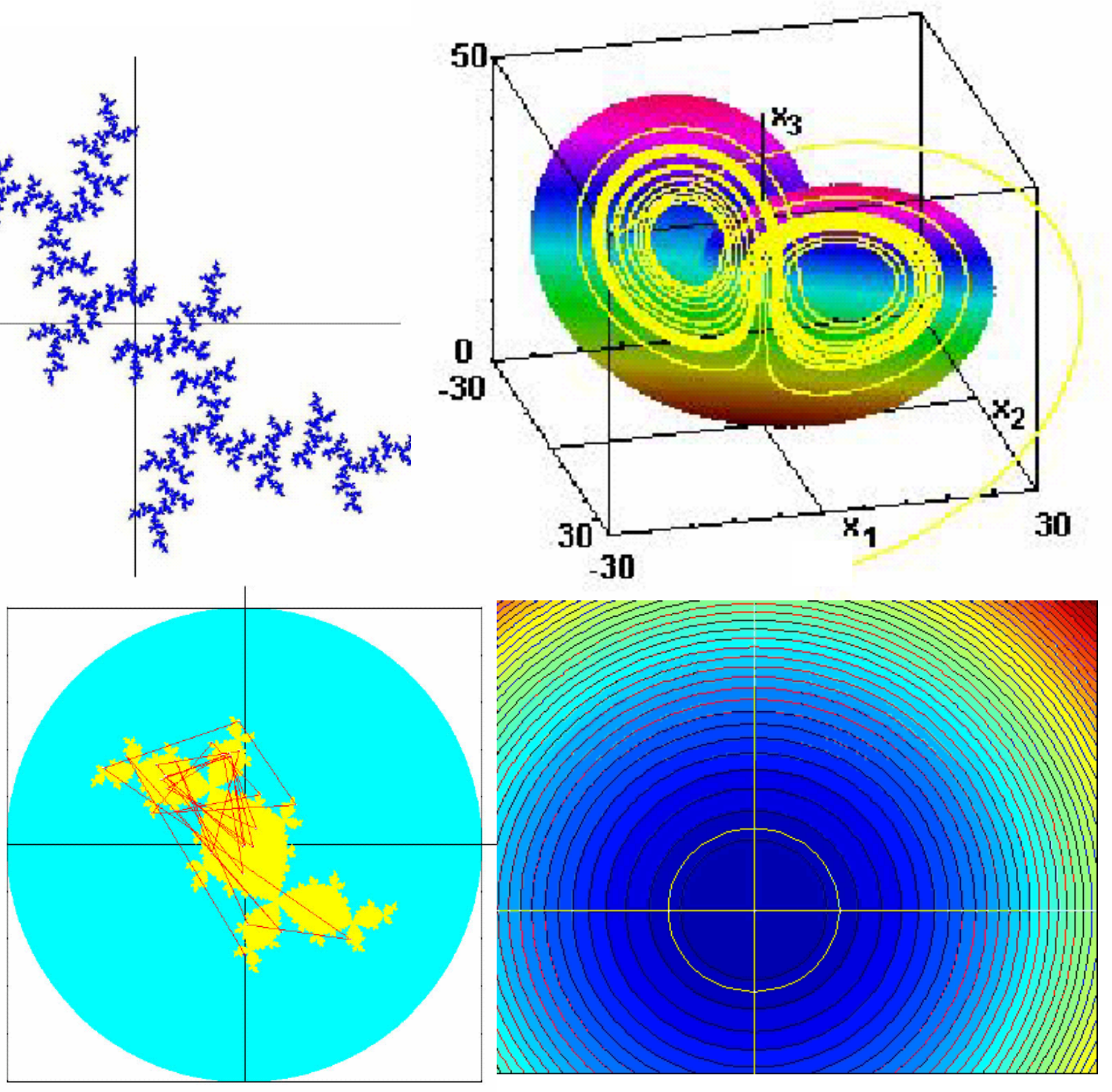


\section{For more information...}

Professor Jean-Pierre Aubin $j \cdot p$.aubin@wanadoo.fr

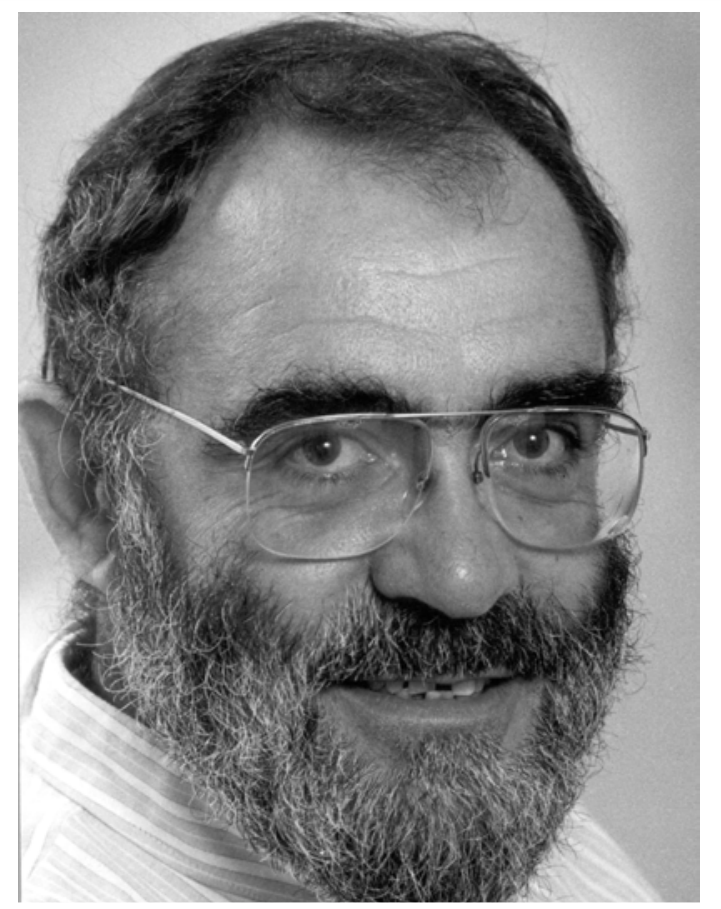

UN I VERSITE PAR IS

DAUPHINE
Professor Nicolas Seube Nicolas.Seube@ensieta.fr

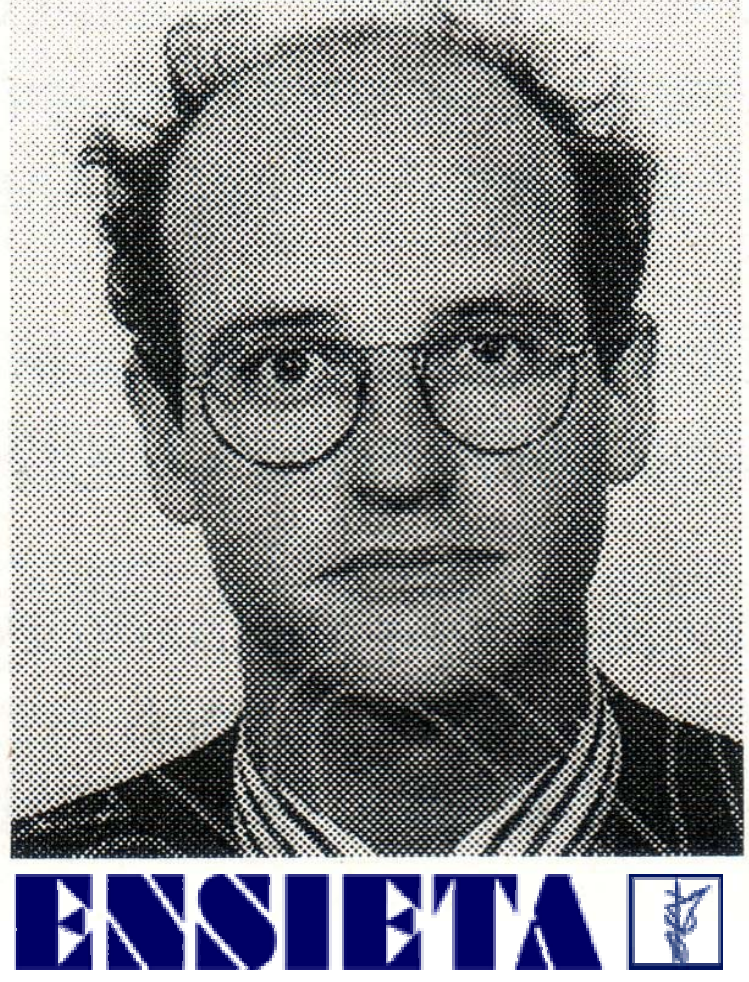

